\title{
Physical modeling of the Bazhenov formation in combination with CSEM and seismic methods
}

\author{
V.V. Ananyev ${ }^{1}$, G.S. Grigoryev ${ }^{2}$,G.D. Gorelik ${ }^{3}$ \\ ${ }^{1}$ Saint-Petersburg Mining University, Department of Geophysical and Geochemical Methods of \\ Prospecting and Exploration of Mineral Deposits, St Petersburg, Russia \\ ${ }^{2}$ Department Manager, Gazprom Neft, Russian Federation,, Saint Petersburg, Russia \\ ${ }^{3}$ Saint-Petersburg Mining University, Department of Geophysical and Geochemical Methods of \\ Prospecting and Exploration of Mineral Deposits, Geological Faculty, St Petersburg, Russia
}

\begin{abstract}
To predict the properties of the Bazhenov formation and to identify the areas in which oil deposits are localized the use of electrical prospecting methods is promising. The main goal of the current research is to evaluate the effectiveness of using Controlled Source Electro Magnetics (CSEM) exploration for the area forecast of the properties of the Bazhenov formation. The main objectives of physical modeling in this research are: Creation of an isotropic physical model corresponding to the electrical and elastic parameters of the section of the studied area, and performing 3D modeling on it with survey parameters that reproduce real seismic and CSEM surveys To convince that the contrast of the elastic properties and the ratio of the resistivity of the target interval of the Bazhenov formation and overlying rocks in the created physical model corresponds to the real petrophysical characteristics of the section of the studied area; Obtaining initial data for testing algorithms for synchronous inversion of seismic + electric and testing various approaches to processing and interpretation of CSEM and 3D seismic data.
\end{abstract}

\section{Introduction}

The oil and gas potential of non-traditional sources has not been estimated in Russia yet, but it far exceeds the volumes of developed oil and gas fields [1].

Works on physical modeling include the creation of an isotropic physical model corresponding in the electrical and elastic parameters to the section of the studied area. It was planned to use sheet technical rubber as the material for the model of the Bazhenov formation (BF) layer. The material for creating the conductive inhomogeneities had to be selected on the basis of field tests of electrical and elastic properties. The model is created based on the conditions for modeling in an electrolytic tank available in Saint Petersburg State University with a size of at least $4 * 4 * 0.4 \mathrm{~m}$, with a scale factor of 1:10 000 .

The choice of an electrolytic tank as the basis for creating a physical model and conducting physical modeling of electromagnetic soundings is determined by the technical feasibility of measuring the electric and magnetic fields of the source used, i.e., a horizontal electric dipole (line) in the frequency range determined by the required scaling factor [2]. The advantages of tank modeling include the ability to smoothly change the 
electrical conductivity of the containing layer and its power and the absence of problems with ensuring electrical contact between the containing structure and electrodes when modeling electric dipoles.

\section{Creating physical model}

The main criterion during choosing materials for modeling was the preservation of the relations of the electrical and elastic properties of the layers during the transition from nature to the model. The basic model consists of 4 layers and in general terms corresponds to the geological section of the study area from top to bottom: 1) sedimentary bed, 2) Bazhenov formation, 3) Jurassic sediments, 4) high-speed basement.

A well-conducting sedimentary bed is modeled in the tank by a layer of electrolyte close to saturation with a $\mathrm{NaCl}$ solution, which, in the range of room temperatures and depending on the concentration, has a resistivity in the range $0.04-0.05 \mathrm{Ohm} * \mathrm{~m}$, and an elastic wave propagation velocity of about $1900 \mathrm{~m} / \mathrm{s}$ [11]. Materials for the model were selected based on the relationship of their electrical and elastic properties to the properties of the first layer.

The second layer, the Bazhenov formation, has a thickness of no more than $50 \mathrm{~m}$ and is a horizon that is sustained in terms of the scale of the studied licensed area and overlies at a depth of about $2300 \mathrm{~m} \mathrm{[3]}$. The rocks of the Bazhenov formation are characterized by elastic wave velocities lower than the overlying rocks, and a noticeable anisotropy of electrical properties - the transverse resistance can exceed the longitudinal resistance by two orders of magnitude [4]. At the stage of preparation of the work, it was decided to simulate the BF with a layer of sheet technical rubber. Rubber is an insulator; however, for modeling purposes, only its transverse resistance can be taken into account, since the longitudinal resistance, due to the low thickness of the layer, should not have any noticeable effect on the results of electromagnetic soundings (the longitudinal conductivity of the $\mathrm{BF}$ is no more than $1 / 500$ of the longitudinal conductivity of the overlying sediments). The transverse resistance of the BS is two orders of magnitude higher than the resistance of the sedimentary bed. Therefore, the representation of this layer during simulation by an insulator is justified.

At the same time, the speed of elastic waves in rubber is less than the speed in electrolyte, which allows, in accordance with the research objective, to provide a contrast of the elastic properties of the target interval (Bazhenov formation) and overlying rocks in the created physical model, corresponding to real petrophysical characteristics of the section of the studied area.

A separate issue is modeling of conductive inhomogeneities in the BF. The possibility of using conductive rubbers used in electrical engineering for this purpose was considered. Unfortunately, measurements showed that the surface of rubber, even electrically conductive, is poorly wetted by liquids (both water and electrolyte), which is an obstacle to the passage of electric current through the surface. As a result, the effective resistivity of the layer of conductive rubber in the electrolyte is too high, which does not allow to provide the required similarity in electrical properties. It was decided to create heterogeneity to change the lateral resistance of the rubber layer using a mesh of holes.

Underlying BF Jurassic deposits, in accordance with the electric logging data, have a rather low resistivity, comparable with the resistivity of the sediment bed [5]. Rubber layers should have reliable placement on the Jurassic rocks layer, while at the same time maintaining a high degree of saturation with the tank electrolyte, which determines the resulting resistivity [6]. Variants of porous ceramics, aerated concrete blocks, and sea pebbles were considered; the final choice was made in favor of a geogrid frame with a mesh size of $210 \mathrm{~mm}$. 
At the base of the section, it overlies a dense high-speed basement with a sufficiently high resistivity, which allows it to be considered an insulator. The basement will be modeled with concrete blocks in the tank.

Data of the electrical and elastic properties of the materials selected to build the model are summarized in Table 1.

Using the selected materials, an isotropic physical model was created, corresponding in electrical and elastic parameters to the section of the studied area. The model diagram is shown in Figure 1, and a section of the model along line $\mathrm{C} 1$ with the designation of the selected materials is shown in Figure 2. The size of the area of simulation of the seismic survey is $1 * 1 \mathrm{~m}$, the size of the region studied by the CSEM is $1.4 * 1.4 \mathrm{~m}$.

Table 1. Data of the elastic and the electrical properties of the materials

\begin{tabular}{llllll}
\hline № & Material & $\begin{array}{l}\text { Specific } \\
\text { resistivity in } \\
\text { model, } \\
\text { Ohm*m }\end{array}$ & $\begin{array}{l}\text { Determined } \\
\text { specific } \\
\text { resistivity, } \\
\text { Ohm*m }\end{array}$ & Speed, m/s & $\begin{array}{l}\text { Determined } \\
\text { speed, } \mathrm{m} / \mathrm{s}\end{array}$ \\
\hline 1 & 0.05 & 4 & 1930 & 3280 \\
2 & $\begin{array}{l}\text { Electrolyte } \\
\text { (saturated NaCl } \\
\text { solution) }\end{array}$ & isolator & isolator & 1530 & 2600 \\
Rubber & Electrolyte with & 0.05 & 4 & 1930 & 3280 \\
4 & $\begin{array}{l}\text { geonet } \\
\text { Concrete }\end{array}$ & isolator & Isolator & 3500 & 5950 \\
5 & $\begin{array}{l}\text { Rubber with } \\
\text { regular mesh } \\
\text { holes }\end{array}$ & 0.75 & 60 & 1530 & 2600 \\
\hline
\end{tabular}
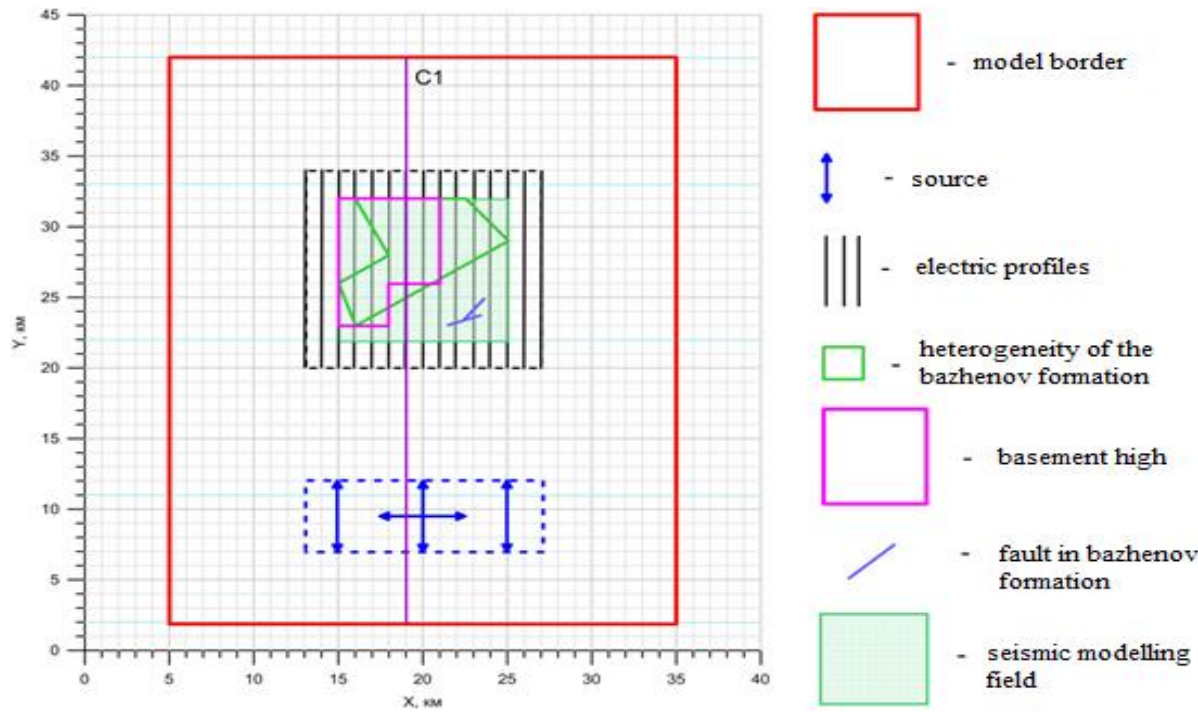

Fig. 1. The scheme of the physical model. The scales along the $\mathrm{X}$ and $\mathrm{Y}$ axes correspond to the dimensions in kind. 
C1

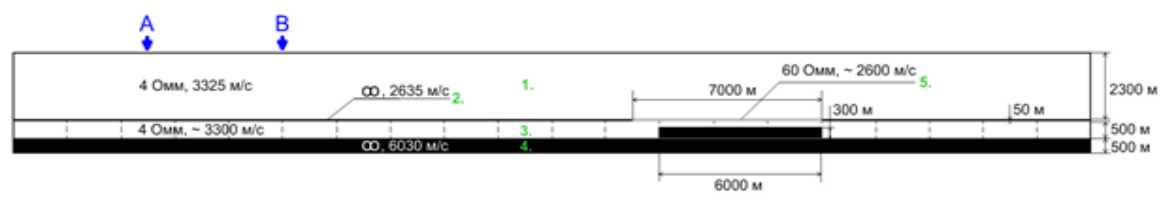

Materials in physical model:

1. Electrolyte(saturated $\mathrm{NaCl}$ solution) $-0.05 \mathrm{Ohmm}, 1930 \mathrm{~m} / \mathrm{s}$

2. Rubber - isolator, $1530 \mathrm{~m} / \mathrm{s}$

3. Electrolyte with geonet $-0.05 \mathrm{Ohmm}, 1900 \mathrm{~m} / \mathrm{s}$

4. Concrete - isolator, $3500 \mathrm{~m} / \mathrm{s}$

5. Rubber with regular mesh holes $-0.75 \mathrm{Ohmm}, 1500 \mathrm{~m} / \mathrm{s}$

Fig. 2. Section of the model along line $\mathrm{C} 1$ with the designation of materials and their properties.

\section{The results of the electromagnetic and seismic modeling}

The plots of various resistivities and phases depending on frequency along all profiles have a similar structure. The main influence on the measured electromagnetic field in the area under study is exerted by the conductive anomaly in the BF. For example, Figure 3 shows the sounding curves and profile curves along profile 12 .
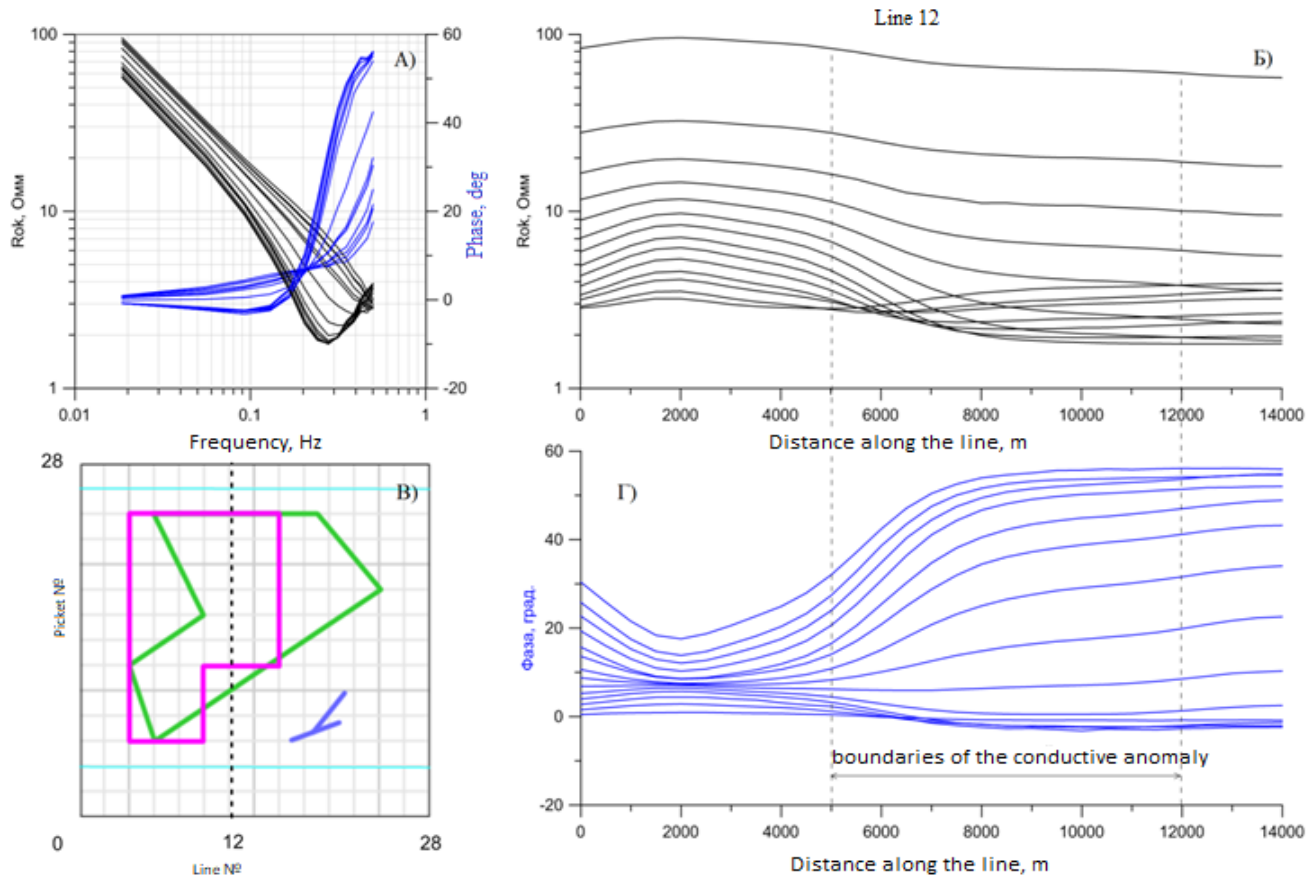

Fig. 3. a) A set of curves of apparent resistivity (black color) and phase (blue color) for profile 12; b) The contours of apparent resistivity; c) The position of the profile 12 on the diagram of the working area of the electromagnetics; d) Isolines of the impedance phase.

When moving from the beginning of the profile to the side of the conductive anomaly, the minimum on the apparent resistivity and phase curves shift toward low frequencies, the values of $\rho_{\mathrm{k}}$ and phase decrease at low frequencies, and an increase at high frequencies occurs. This transition occurs in a fairly wide range of distances. A characteristic feature is 
that when the receiver leaves the anomaly, the values of $\rho_{\mathrm{k}}$ and phase do not return to the level that they had at the beginning of the profile.

On the profile curves, an increase in the apparent resistance and a decrease in the phase in the area of the fourth pickets (2000 $\mathrm{m}$ from the beginning of the profile) are noticeable. This effect can be associated with the passage of the receiver through the joint of the rubber sheets modeling the Bazhenov formation.

The effect of three-dimensional inhomogeneitiescan be most clearly observed on the maps of isolines $\rho_{\mathrm{k}}$ and phases built for different frequencies. The contour maps for 5 harmonics $(0.056 \mathrm{~Hz}, 0.167 \mathrm{~Hz}, 0.278 \mathrm{~Hz}, 0.389 \mathrm{~Hz}, 0.501 \mathrm{~Hz})$ are presented in Figure 4. It should be noted that due to the large differences in the intervals of variation of the effective parameters at different frequencies, the color scales in the figures have different limits [7].

A linear anomaly is observed in the area of the fourth pickets on all isoline maps (coordinate $\mathrm{Y}=2000 \mathrm{~m}$ ), which is also visible on the plots of various resistivities and phases depending on frequency, probably related to the influence of the junction of rubber sheets simulating BF. The presence of conductive heterogeneity in the BF is manifested by an anomaly of low resistance at low frequencies and increased resistance at high frequencies at $\mathrm{Y}>6000 \mathrm{~m}$. The same pattern (transition from low to high) is observed for the phase. The direction of the isolines, on the whole, correlates with the boundary of the conducting inhomogeneity but does not completely repeat it. In the area of 8-16 profiles and 2-6 pickets, an anomaly of high resistance is observed, which can probably be associated with a closely located protrusion of inhomogeneities.

However, it does not seem possible to separate the effect of base heterogeneity and conductive heterogeneity in the BF layer at the level of effective parameters. The presence of inhomogeneities in the model leads to a redistribution of the exciting electric currents and to a change in the values of the effective resistivity and impedance phase over the model and in its immediate vicinity, but the distribution of effective parameters in the plane does not necessarily repeat the contours of the inhomogeneities, since the interaction of the electromagnetic field with the structure has a complex character.

One of the objectives of the project is to compare the sensitivity of the CSEM method to low-power formations of high resistivity during measurements on the axis and in the equatorial zone in order to select the optimal array for fieldwork [8]. To solve this problem, physical modeling of measurements was performed with an equatorial array over the $3 \mathrm{D}$ model according to one of the profiles in the CSEM survey work area (profile 12), as well as 2D mathematical modeling for the model, which is a continuation in the $\mathrm{X}$ direction of the $3 \mathrm{D}$ model section along the 12 th profile.

The span in physical modeling with an equatorial setup was $1.2 \mathrm{~m}$, as in measurements with an axial setup. A comparison of the results for the axial and equatorial setup is shown in Figure 5.

Although the magnitude of the anomaly for the three-dimensional model is smaller than for the two-dimensional, it can be seen that in any case the axial setup is more sensitive to resistivity variations than the equatorial one, and, therefore, should be used primarily during field works. 

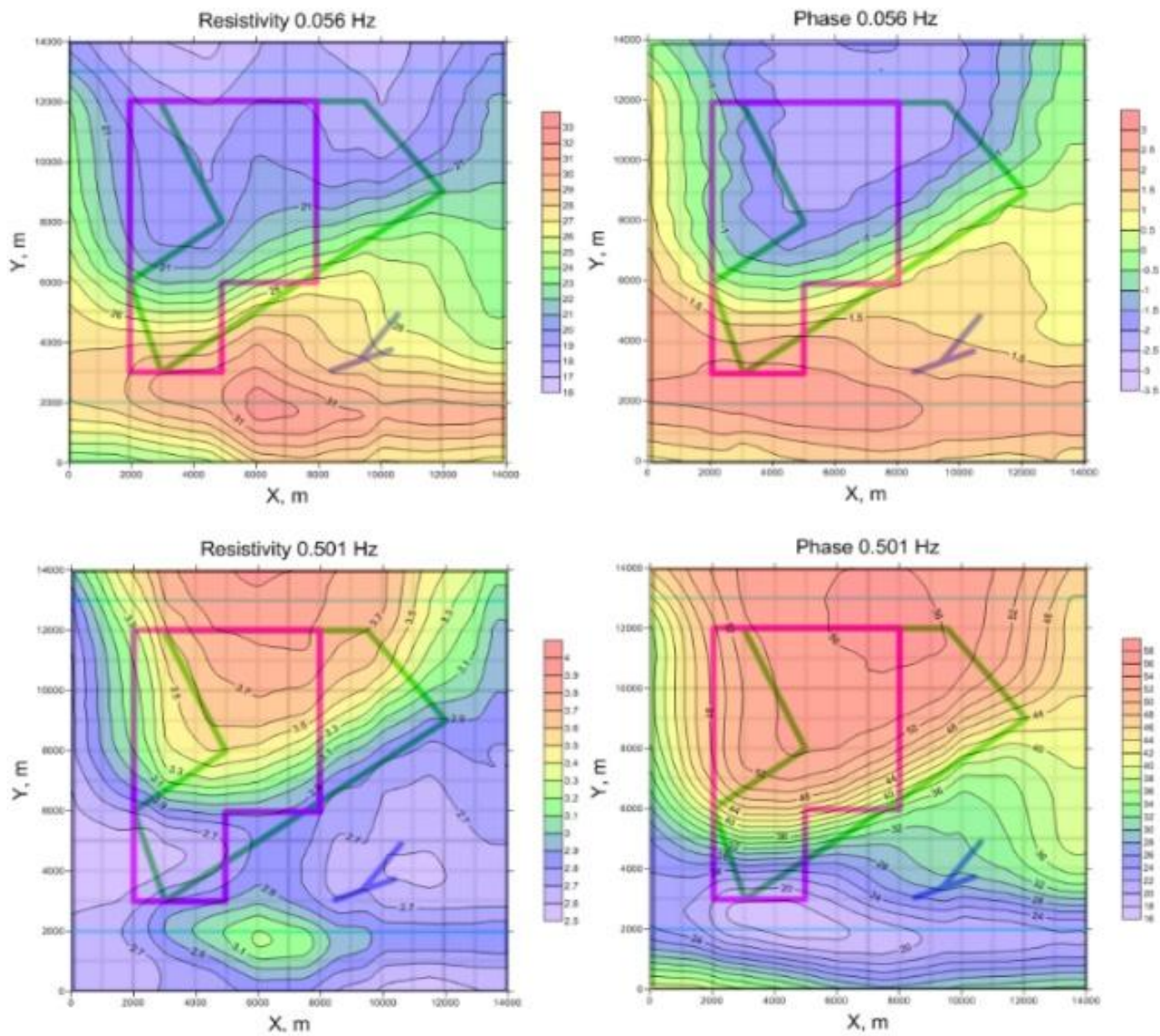

Fig. 4. Maps of isolines of apparent resistance and phase at different frequencies.
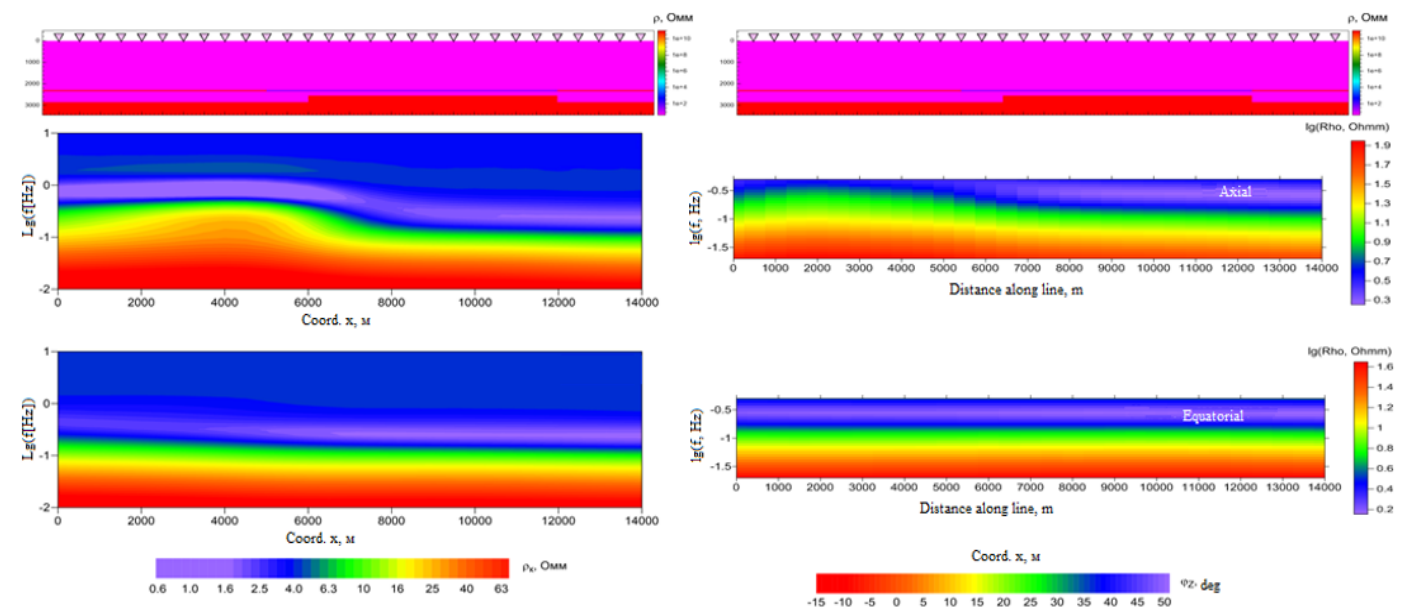

Fig. 5.Pseudodepth plots of apparent resistance for axial and equatorial installations. Left - 2D mathematical modeling, right - 3D physical modeling. 
Data of a good signal-to-noise ratio were obtained as a result of seismic modeling [9], highlighting the target research objects: reflections from the Bazhenov formation layer, "fault" zone, an anomaly in the Bazhenov formation, reflections from the basement (Figures 6 and 7).

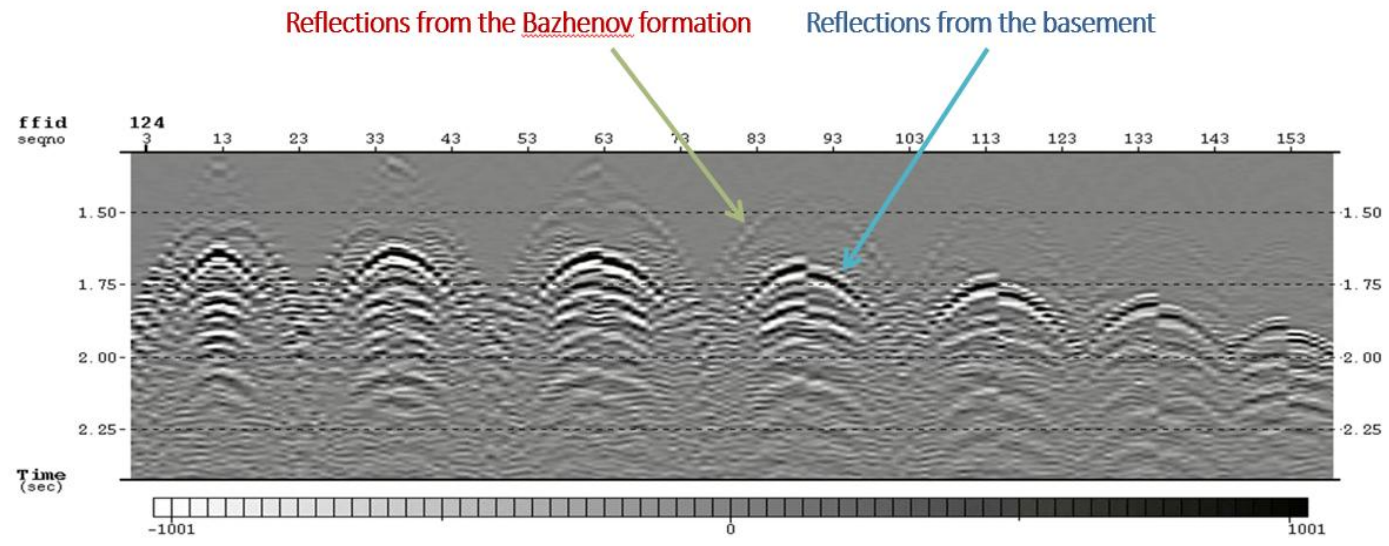

Fig. 6. Seismogram obtained as a result of physical modeling.

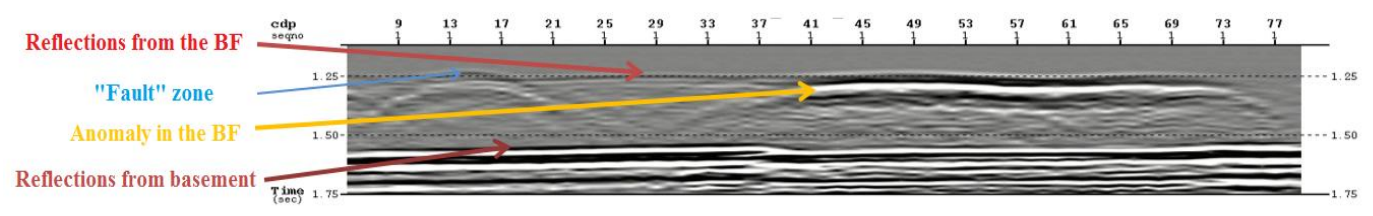

Fig. 7. TWT section with a detailed description.

\section{Conclusion}

Big amount of data was collected in open sources as a result of the study. An assessment of the quality of the data obtained was made. The choice of materials for creating a physical model was made, field measurements of their elastic and electrical properties were carried out. An isotropic physical model was created based on the selected materials, which, taking into account scaling and similarity conditions, corresponds in electrical and elastic parameters to the section of the studied area [10].

A physical simulation of CSEM seismic and soundings was performed with the configuration and operating parameters of the arrays, reproducing, taking into account scaling, the parameters used in real field surveys in the study area. An array of high-quality seismic and electromagnetic data has been obtained, which can be used to test algorithms for synchronous inversion of the results of seismic + electric results and to test various approaches to the processing and interpretation of CSEM and 3D seismic data. The inhomogeneities of the physical model are clearly manifested in the data of both seismic and electromagnetic modeling. In the case of pronounced three-dimensionality of the medium during CSEM sounding, maps and graphs of effective parameters give only an approximate idea of the shape and position of geoelectricinhomogeneities.

To restore the geology aspects of the structure according to CSEM data, it is necessary to use seismic information in the form of setting structural boundaries or a joint inversion of seismic and electromagnetic data. 


\section{References}

1. Y.V. Nefedov. EAGE The Arctic shelf and hard to recover oil reserves as alternative development of Russian resource base, 1-7 (2018).

2. O. Putikov, M Kholmyanski, G. Ivanov, N. Senchina. Chemie der Erde Application of geoelectrochemical method for exploration of petroleum fields on the Arctic shelf $\mathbf{8 0}$ (3), (2020).

3. A.I. Ingerov, I. Mendrii, A.Lozoviy. 4th European Meeting of Environmental and Engineering Geophysics Integrated field surveys with complex of electroprospecting methods using broadband multifunction EM instruments, 12-15 (2018).

4. Y.V. Braduchan, F.G. Gurari, V.A. Zakharov, Bazhenov horizon of Western Siberia. Moscow: Nauka (1986).

5. A.I. Ingerov, I.A. Lozovoy, A.L. Mendriy, Naukovyi Visnyk Natsionalnoho Hirnychoho UniversytetuDifferential curves of induction electromagnetic soundings with natural sources for structural mapping in oil and gas, 42-48 (2015).

6. O.M Prischepa, Y.V. Nefedov, O.E. Kochneva. Periodico Tche Quimica Raw material base of hard-to-extract oil reserves of Russia34(17), 915-923 (2020).

7. M.M. Saitgaleyev, N.P.Senchina, J.A. Sokolova. Marine TechnologiesApplication of the method of ion-selective electrodes in exploration work on the sea shelf, 144-154 (2019)

8. N. Senchina. Notes of the Mining Institute Analysis of the correlation between the results of measuring the natural electric field and the contents of chemical elements (by the example of objects of the Middle Urals)141, (2015)

9. A.P. Sysoev, G.D. Gorelik. Russian Geology and Geophysics Parametric method of compensation for near-surface heterogeneity in processing CDP data 58(6), 763-768 (2017)

10. A.A. Vashkevich, K.V. Strizhnev, S.I. Chekmarev, D.E. Zagranovskaya, A.S. Bochkov, V.V Zhukov, Yu. Zuikova,.N.F.Skopenko. Oil industry Experience in integrating the methods of potential fields and areal geochemical studies in order to plan geological exploration in poorly studied areas of the Bazhenov formation12, 3235 (2016).

11. G.S.K Wong,.S. Zhu. J. Acoust. Soc. Am. Speed of sound in seawater as a function of salinity, temperature, and pressure97(3), 1732-1736 (1995). 